\title{
BMJ Open Collective leadership and safety cultures (Co-Lead): protocol for a mixed-methods pilot evaluation of the impact of a co-designed collective leadership intervention on team performance and safety culture in a hospital group in Ireland
}

Eilish McAuliffe, ${ }^{1}$ Aoife De Brún, ${ }^{1}$ Marie Ward, ${ }^{1}$ Marie O'Shea, ${ }^{1}$ Una Cunningham,,${ }^{1,2}$ Róisín O'Donovan, ${ }^{1}$ Sinead McGinley, ${ }^{1}$ John Fitzsimons, ${ }^{3,4}$ Siobhán Corrigan, ${ }^{5}$ Nick McDonald ${ }^{5}$

To cite: McAuliffe E, De Brún A, Ward $\mathrm{M}$, et al. Collective leadership and safety cultures (Co-Lead): protocol for a mixedmethods pilot evaluation of the impact of a

co-designed collective leadership intervention on team performance and safety culture in a hospital group in Ireland. BMJ Open 2017;7:e017569. doi:10.1136/ bmjopen-2017-017569

- Prepublication history for this paper is available online. To view these files, please visit the journal online (http://dx.doi. org/10.1136/bmjopen-2017017569).

Received 8 May 2017 Revised 28 July 2017 Accepted 16 August 2017

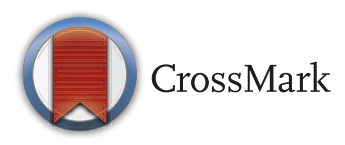

For numbered affiliations see end of article.

Correspondence to Dr Aoife De Brún; aoife.debrun@ucd.ie,debruna@ tcd.ie

\section{ABSTRACT}

Introduction There is accumulating evidence implicating the role of leadership in system failures that have resulted in a range of errors in healthcare, from misdiagnoses to failures to recognise and respond to patient deterioration. This has led to concerns about traditional hierarchical leadership structures and created an interest in the development of collective ways of working that distribute leadership roles and responsibilities across team members. Such collective leadership approaches have been associated with improved team performance and staff engagement. This research seeks to improve our understanding of collective leadership by addressing two specific issues: (1) Does collective leadership emerge organically (and in what forms) in a newly networked structure? and (2) Is it possible to design and implement collective leadership interventions that enable teams to collectively improve team performance and patient safety? Methods and analysis The first phase will include a social network analysis, using an online survey and semistructured interviews at three time points over 12 months, to document the frequency of contact and collaboration between senior hospital management staff in a recently configured hospital group. This study will explore how the network of 11 hospitals is operating and will assess whether collective leadership emerges organically. Second, collective leadership interventions will be co-designed during a series of workshops with healthcare staff, researchers and patient representatives, and then implemented and evaluated with four healthcare teams within the hospital network. A mixed-methods evaluation will explore the impact of the intervention on team effectiveness and team performance indicators to assess whether the intervention is suitable for wider rollout and evaluation across the hospital group.

Ethics and dissemination Favourable ethical opinion has been received from the University College Dublin Research Ethics Committee (HREC-LS-16-116397/LS-16-
Strengths and limitations of this study

The study is unique in that it explores if health system reconfiguration on its own enables leaders to work more collectively and collaboratively across hospitals.

- A key strength of this research is that the engagement of healthcare teams and patients in the co-design of the collective leadership intervention will ensure that the intervention is designed collaboratively, thus ensuring its relevance.

- Given it is not feasible to test the intervention in all types of healthcare teams, the pilot will evaluate the intervention on four selected teams that differ in terms of size, specialty and time established.

20). Results will be disseminated via publication in peerreviewed journals, national and international conferences, and to relevant stakeholders and interest groups.

\section{BACKGROUND}

Health systems worldwide are undergoing significant reform and change, motivated by changing political environments, technological and medical advancements, and increased burden on healthcare services due to ageing populations cost. ${ }^{1}$ The Irish health system has experienced significant changes in the past 30 years, with a period of substantial reform since the 1990s. ${ }^{2}$ However, these organisational changes have not typically been driven by evidence-based research of what works, and there has been very little measurement of the resulting impact on 
leadership, healthcare delivery and patient safety. Such reconfigurations have led to considerable changes in leadership titles and responsibilities, and often there is insufficient time (and in some cases skills) for leaders to develop in these new positions before the next wave of reform. Arguably, this has undermined the role of leadership and impacted negatively on the performance of staff. In addition, there has been an inconsistent approach to leadership development; while there are examples of excellent leaders, there is also a growing body of evidence of how poor or absent leadership has contributed to system failures that have resulted in a range of errors, from misdiagnoses to failure to recognise and respond to patient deterioration. ${ }^{2-4}$ The most recent reorganisation of the Irish health service into hospital groups aims to encourage collaborative working across boundaries to reduce fragmentation and achieve better integration between primary and secondary care. ${ }^{5}$ This provides a valuable opportunity to explore leadership requirements in this new environment of collaboration and to develop and evaluate an approach to leadership that advocates the sharing of leadership responsibilities and roles.

A number of adverse events over the past decade prompted the establishment of the Commission on Patient Safety and Quality Assurance in 2007. ${ }^{3}$ The work of the Commission acknowledged serious patient safety-related shortfalls in the current system, such as poor communication processes, weak governance structures and poor working relationships between clinicians and management. ${ }^{3}$ Leadership has also been emphasised as playing a crucial role in healthcare and safety cultures. A recent review of evidence of leadership in healthcare by West $e t a t^{6}$ identified one of the key challenges for healthcare organisations as promoting cultures that ensure the delivery of continuously improving high-quality, safe and compassionate healthcare. The review emphasised the importance of leadership as 'the most influential factor' in shaping organisational culture, asserting the necessity of effective leadership development strategies to enable health services improvement. ${ }^{6}$

Research has indicated that effective leadership in a clinical setting is associated with improved quality and safety. ${ }^{7}$ Additionally, where health staff report they are well-led and have high levels of satisfaction with their immediate supervisors, patients report that they, in turn, are treated with respect, care and compassion. ${ }^{8}$ This underlines the link between effective leadership practices, staff satisfaction and positive patient outcomes. Staff engagement also appears to be greater in healthcare organisations where staff members feel involved. In an investigation of organisational factors, culture, leadership, staff wellbeing and patient safety in eight UK healthcare organisations, McKee found that the best performing hospitals were characterised by high staff engagement in decision-making and widely distributed leadership. ${ }^{9}$

These findings highlight the need for a more inclusive approach to leadership, one that is typified by shared responsibility and accountability and a focus on collective impact rather than individual achievement. There is a growing interest in shared or collective leadership styles, which may be defined broadly as 'an emergent and dynamic team phenomenon whereby leadership roles and influences are distributed among team members'. ${ }^{10}$ Such approaches are characterised by distributed roles and responsibilities and the selective utilisation of the skills and expertise of individuals, as required by the task or situation at hand. ${ }^{11}$ Recent research indicates that, across sectors, shared leadership in teams predicts team effectiveness. ${ }^{10} 12$ West $e t a l^{13}$ differentiate collective leadership cultures from traditional hierarchical approaches by describing collective leadership as occurring when 'cultures, responsibility and accountability function simultaneously at both individual and collective levels'. In contrast, traditional 'command and control' cultures can 'invite the displacement of responsibility and accountability onto a single individual, leading to scapegoating and a climate of fear of failure rather than an appetite for innovation'. ${ }^{13}$

The recent reorganisation of the Irish health system into hospital groups provides a timely opportunity to explore the concept of collective leadership and its potential to impact on healthcare quality and safety. This research will draw on emerging theories of collective leadership ${ }^{10}{ }^{12}$ emphasising the leadership capacity of teams rather than individuals. The central hypothesis underpinning the research programme is that collective leadership will improve healthcare team performance and staff engagement, which will lead to an enhanced safety culture and improvements in quality and safety. This hypothesis is based on previous studies' findings that shared leadership predicts team effectiveness, ${ }^{10}{ }^{12}$ and on the previously verified association between effective leadership and improved quality and safety. ${ }^{7}$ The first phase of the research programme will explore whether collective leadership emerges organically among senior management following reconfiguration of 11 hospitals into a network, and what forms this may take. The next phase of the work involves developing an understanding of the leadership requirements for effective team working at the service delivery level to ensure safe care for patients, by working with healthcare staff to co-design an intervention to develop collective leadership in healthcare teams. This intervention will then be implemented with four healthcare teams in the hospital network and evaluated to empirically test the impact of the intervention on team performance and patient safety cultures.

\section{METHODS}

The first phase of this research involves a top-down approach using a cohort of senior managers to explore whether collective leadership emerges organically following the reconfiguration of 11 hospitals into a hospital network. In the next phase, a bottom-up approach will be adopted, working with front-line healthcare staff and patient representatives to co-design a collective leadership 
intervention, which will then be implemented in selected teams and subject to a mixed-methods evaluation.

\section{Context of the research}

This research will be conducted within the Ireland East Hospital Group, the largest of the seven newly established hospital groups in Ireland. The group consists of 11 hospitals in the east of Ireland that range from small specialty hospitals to large acute teaching hospitals. Given that 6 of the 11 hospitals in the group are voluntary and 5 are statutory, funding and operating structures differ between hospitals in the group. Together the hospital group employs over 10000 people and serves a population of 1.1 million people in the region. The rationale for the restructuring of hospitals into groups is summarised as:

The establishment of Hospital Groups and, subsequently, Hospital Trusts, will enable hospitals to provide care in the right way, at the right location. This must be done in a manner that ensures a safe, high quality service for all, maximising and capitalising on the strengths of both larger and smaller hospitals, with best outcomes for patients paramount in every facet of their services. ${ }^{14}$

\section{Phase 1: mapping leadership networks}

The aim of this phase is to map the networks of leadership that develop at the senior management level within the newly established hospital group over a 1-year period. This natural experiment will explore whether collective leadership emerges organically following the reorganisation of the hospitals into a networked structure and will examine factors that facilitate and hinder integrated leadership.

\section{Methods}

This 12-month case study will use a cross-sectional cohort of senior managers across the 11 hospitals in the network and the hospital group management team. Social network analysis (SNA) is the methodological approach that will underpin a variety of data collection methods, including in-depth interviews and online surveys. SNA enables the mapping, measurement and analysis of social relationships between people, teams and organisations. ${ }^{15}$ It facilitates the exploration of patterns and types of relationship between actors (individuals, teams or organisations), where these actors are visually represented in a network map via structural nodes and relationships between these nodes. SNA allows for the analysis of the role and influence of various actors in a network, can characterise and map network relationships, and analyse the structure of a system. ${ }^{16}$ Using the specialised software package UCINET, ${ }^{17}$ researchers can explore how patterns of relationships can operate to facilitate or inhibit communications, actions and capacities. ${ }^{18}$ SNA has been employed in a myriad of healthcare settings, often as a descriptive tool. $^{19}$
SNA is the most appropriate approach as it has the capability to be used both as a descriptive and a diagnostic tool to understand how the network is operating, and provide guidance of where attention needs to focus to improve network operations. It enables the visual depiction of the network map, highlighting areas of the network that are operating effectively and others that may be more isolated or removed from the network. It can be a powerful tool in understanding the structure of the network, and mapping interactions at multiple time points will enable a deeper understanding of how the network interactions develop and evolve over time.

\section{Sample and recruitment}

The chief executive officer/general manager, director of nursing and clinical/medical director in each of the 11 hospitals, as well as the senior management team for the hospital group, will be invited to take part in the SNA $(n=\sim 45)$. They will be asked to opt-in and to register their consent to participate in the study.

\section{Data collection}

Data will be collected over a 12-month period, with network maps produced at three time points: months 1 , 6 and 12. An online survey has been developed using a roster method, which asks each person to provide information on other named individuals in the network based on the specific question asked. The variables of interest in the current study include the frequency of contact and collaboration between network members and understanding who in the network they contact for support in their role. Given that these individuals are all in leadership positions, asking specifically about leadership will not provide insight into how the managers are integrating and using the network. Exploring their levels of contact and collaboration, on the other hand, will provide insight into if and how senior management in the hospital group are integrating and collaborating across sites.

The evaluation of collective leadership presents unique challenges in that collective networks tend to be dynamic, and clusters (and connectivity between clusters and nodes) may be fluid, adapting to changing demands. ${ }^{20}$ Thus, depicting a network map at only one time point may not be very informative. Accordingly, mapping the network at three time points will, to some extent, facilitate our understanding of the dynamic nature of the network, exploring whether (and how) the network structure may change over time. ${ }^{21} 22$

The online survey to collect network data was pilottested using cognitive interviewing with two network members before going live. Minor amendments were made to the survey based on the feedback received.

One-on-one semistructured interviews will also be conducted with a subset of participants who volunteer for interview at each data collection point $(n=\sim 5)$. The qualitative data will provide insight into how the group is operating and will highlight the barriers and facilitators individuals have experienced in collaborating and 


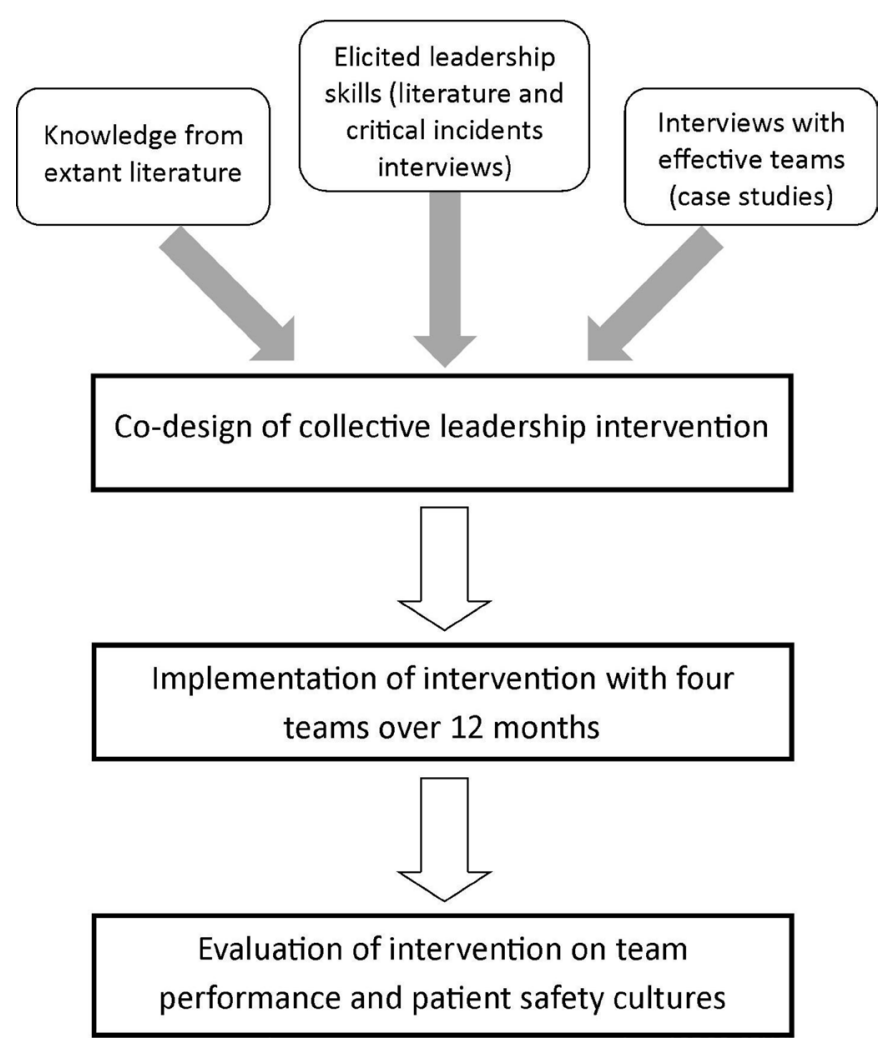

Figure 1 Summary of intervention co-design process and evaluation.

working collectively. The interviews will also allow for the collation of information to generate a database of ongoing collaborations and activities in the group, and will help to inform the analysis and interpretation of the social network maps. Qualitative data will be analysed using thematic analysis. ${ }^{23}$

\section{Phase 2: c-odesign of collective leadership intervention(s)}

While phase 1 of the research will focus on exploring collective leadership at the top level of the hospital group, phase 2 will adopt a bottom-up approach in developing a collective leadership intervention with front-line healthcare staff. Rather than the traditional focus on the individual as the leader, the development process for the intervention will be informed through a service needs-driven, co-designed intervention targeted at team members as co-leaders. The majority of education and development programmes for leaders focus on developing the individual as an autonomous leader. As it becomes increasingly evident that the interdependencies in healthcare require more collective leadership approaches, ${ }^{6} 101213$ there is a need to question the common practice of providing leadership training to a designated leader in isolation from his/her team, as well as reconsidering the content, teaching methods and learning outcomes of leadership programmes. Therefore, this phase of the research aims to develop and test a new model of leadership development, underpinned by the concept of collective leadership. ${ }^{11}$ Our hypothesis, informed by the extant literature on collective leadership, is that collective leadership will lead to effective teamwork, which in turn will impact positively on patient safety. In contrast to traditional approaches that focus development on the individual, this approach will focus on developing the team as a dynamic leadership entity, ensuring that all members understand and develop the capability for leadership.

The task for an intervention co-design team will be to consider how to provide the skills to a team to collectively encourage and support each member of the team to achieve the team's goals. There will be a need for learning to be a dynamic interactive process, and the requirement to shift thinking from the traditional concept of the individual learner, and the knowledge and expertise being embodied in one individual leader, to the notion of group learning and a shared repository of leadership skills.

Due to the variance in types of teams in the hospital group (ie, in terms of size, specialty, time established and so on), it is conceivable that collective leadership may generate improved performance in one type of team more than another. For this reason, this research will select four different team types in the hospital group to help develop, refine and test the co-designed collective leadership intervention. These teams will be diverse in terms of their specialist area, level in the healthcare system, time working together and scope of operation (within site and across site teams). Ensuring different team types are included in the co-design process will help to ensure the intervention is appropriate for various team types and will provide insight into the common leadership needs identified by healthcare teams, as well as exploring whether leadership development needs differ according to team type. In addition to these four teams being involved in the co-design and implementation, it is anticipated that a team of managers from the cohort in phase 1 of the study will also take part as an intervention team.

\section{Methods}

A co-design team will be formed with up to two volunteers from each of the four selected healthcare teams, individuals from the wider healthcare system, the research team, and two patients or patient representatives $(n=16-19)$. A series of six 3-hour co-design workshops will be held over a 6-month period to develop and prioritise the collective leadership intervention content. The intervention components agreed by the co-design team will then be developed into a set of resources, which will be included in the intervention.

The co-design workshops will be informed by a variety of data sources, as illustrated in figure 1. These will include the following:

\section{National and international case studies of effective teams}

Informed by expert opinion, a number of teams and organisations will be invited to take part in interviews to develop a diverse set of case studies to provide insight into how effective teams work and the processes that were enacted or resources provided to enable teams to 
work together effectively. These case studies will include different team types that have been recognised nationally and internationally as successfully delivering and working collaboratively to improve healthcare delivery and patient care. Up to 20 semistructured interviews will be conducted to provide insight into perspectives on effective team working from individuals identified as working on effective teams (both within and outside the hospital group), and those in organisations who have driven successful organisational strategies to enable more effective teams. ${ }^{24}$ Findings emerging from these interviews will be presented to the codesign team to inform the intervention codesign process.

\section{Relevant knowledge from extant literature}

Literature reviews will also be conducted in areas relevant to the research to inform the co-design process. A systematic review to explore collective leadership interventions in healthcare settings will be conducted. Reviews will also explore patient safety literature and literature on effective teamwork.

\section{Factors contributing to or inhibiting effective teamwork (contexts, mechanisms, outcomes)}

Guided by a review of literature on teamwork in healthcare, a series of critical incident interviews ${ }^{24}$ will also be conducted to explore positive and negative experiences of teamwork in depth to understand the context and mechanisms that facilitate and inhibit effective collaborative working in healthcare teams and in healthcare organisations. Critical incidents are incidents or events that are critical to the person's view of a phenomenon or problem. This is a technique that is commonly used for collecting incidents that the respondent feels have been critical to an individual's experience of a job. Once the incident has been recorded, the interviewer uses probing questions to elicit the details of the incident and the respondent's reactions and feelings about the incident. ${ }^{24}$ This approach enables an exploration of the skills, attitudes and behaviours influencing his/her own or other actors' behaviours, which ultimately helps to identify skills gaps or the need for behaviour or attitude change. It is anticipated that up to 30 participants will be recruited to the study to collect information on $\sim 50$ critical incidents. Sampling will include a broad range of participants, in terms of level of experience, team type and roles. Data from critical incident interviews will be used to understand the contexts, mechanisms and outcomes related to factors that contribute to or inhibit effective team working.

\section{Phase 3: implementation and evaluation of the codesigned intervention}

The co-designed intervention will be implemented over a 1-year period and evaluated with the selected teams. Ensuring different team types are included in the evaluation will test whether the intervention is appropriate for all team types or whether it may need further refinement or adaptation before wider roll-out across the hospital group. Workshops will be held with each intervention team to discuss shared goals and identify the leadership skills and competencies the teams will need to develop in order to achieve their goals. During the initial workshops, the intervention teams will be asked to examine the evaluation measures, including key performance indicators, quality performance indicators and safety performance indicators, most meaningful to their performance as a team. Teams will self-select relevant indicators that they seek to improve through the collective leadership intervention. These evaluation measures will be assessed at baseline and postintervention to explore the impact of the intervention. The teams' learning preferences will be assessed, and learning events will be mapped to an agreed work plan with clearly identified objectives, timelines and outputs. It is anticipated that learning events may take a variety of different formats, including online modules, webinars, face-to-face didactic sessions, face-to-face and online discussions, and questions and answers with facilitators or similar teams from other organisations.

\section{Methods}

As collective leadership is more than the sum of individual role-taking, it will need to be assessed accordingly. ${ }^{25}$ Assessment of the whole team is the most appropriate method to assess the impact of the intervention on performance. The four healthcare teams identified will form the cohort for testing the impact of the leadership intervention on the leadership skills, actions and behaviours of the participants. Teams will be invited to take part in a structured programme over a 12-month period with the collective leadership intervention tailored to meet the needs of each team. Each learning event will be exclusive to the particular team, meaning that programmes will run in parallel for the various teams. While there may be considerable overlap in the material and content, the experience of learning within a primary reference team is an important component of the collective leadership approach. Learning events will take place primarily in the hospitals where the teams are located, and rotating venues where teams are spread across sites.

Selection of appropriate and robust evaluation methods and measures to assess the intervention will be informed by the co-design team and through the initial workshops with the intervention teams to prioritise goals and targets in relation to team performance and meaningful metrics. For instance, the research team will present the co-design team with a number of options of validated and reliable scales related to general team performance and safety culture. Discussions regarding the relevance of various measures for evaluation will focus on the aims and objectives of the research and on the finalised intervention content, with the intention that the evaluation measures should align as closely as possible with the final intervention content. In addition to those that may be identified during these processes, the Aston Team Performance Inventory (ATPI) or the Team Climate Inventory (TCI) will be used to measure team performance at baseline and postintervention. ${ }^{26}{ }^{27}$ These scales would provide 
a comprehensive measure of team performance and results presented in a report giving feedback about how the team is performing and showing levels of agreement between team members about the way in which the team is performing. ${ }^{26}{ }^{27}$ The co-design team will select what they deem the most appropriate measure.

Multiple forms of data will be required to enable a thorough and rich assessment of various aspects of team performance and patient safety to understand the potential impact of the intervention. ${ }^{28}$ Given the challenges in interpreting cause and effect of interventions in complex systems, it is important to collect data that can provide insight into the experiences of those affected by an intervention, and that explore both the intended and unintended consequences. ${ }^{29} 30$ Therefore, alongside the quantitative team performance data, qualitative data will be collected at baseline and postintervention from members of the four healthcare teams to explore perceptions of how the team operates and team processes, and to understand if and how the intervention may impact on team working, performance and collective leadership behaviours. Semistructured interviews will be conducted with participants from each of the four teams to explore the baseline operation of the team and compare that with team members' experiences postintervention to understand what, if any, impact the intervention has had on team processes and effectiveness. The interview guide was designed to provide a broad overview of teams' processes and current ways of working, team dynamics and safety culture. Questions relate to working relationships, communication, problem-solving, psychological safety and openness, trust, satisfaction, and team safety performance and measurement. The interview guide was pilot-tested with healthcare professionals working in teams, but not involved in the current phase of the work. The guide was refined iteratively through discussion in the research team.

\section{Patient/public and stakeholder involvement}

We will engage patient representatives as members of the co-design team and envisage them playing an active role in the development of the collective leadership intervention. The personal experience of healthcare of patients/carers/ members of the public, their experience of patient safety incidents, as well as their experience as members of investigation teams are invaluable in designing the intervention. Patient representatives on the team will also be part of all analysis and dissemination meetings and workshops, and we plan to engage them as coauthors in the publications arising from this research. In addition, two patient representatives will be invited to join the Advisory Group.

\section{ETHICS AND DISSEMINATION}

Favourable ethical opinion for the research has been obtained from the University College Dublin Research Ethics Committee (ref: HREC-LS-16-116397/LS-1620). This research began in December 2015 and will be completed by April 2021.
Research findings will be shared as they become available at the end of each phase of the research. The research team will present findings at national and international conferences, to public/patient interest groups, and publish in peer-reviewed journals. The Co-Lead research programme will have a dedicated website on which all resources and research findings will be posted as they become available. Materials and resources will also be made available through the Irish Health Service Executive's online repository.

\section{DISCUSSION}

This research programme aims to explore, on the one hand, whether collective leadership emerges organically following the configuration of a hospital network, and on the other, whether it can be developed in existing and new teams. The study is unique in that it explores if reconfiguration on its own enables leaders to work more collectively and collaboratively across hospitals, while at the same time co-designing and testing a tailored intervention to try and develop collective leadership skills in healthcare teams. This work will significantly contribute to the emerging theory and developing evidence base around collective leadership in healthcare settings.

The first phase of this work will employ SNA to study the level of contact and collaboration among senior management across the top level of the hospital network. The results of this phase will provide insight into whether collective leadership emerges organically following the configuration of 11 hospitals into a networked structure, or whether the network requires further support or intervention to enhance integration and collaboration.

The next phase will then adopt a bottom-up, needsdriven approach by working with front-line staff to co-design a collective leadership intervention. Given the co-designed nature of the intervention, it is impossible to prespecify what the intervention will include or how exactly it will be delivered. ${ }^{31}$ However, the approach will ensure that the intervention components are grounded in the needs and real-world experiences of healthcare staff. In co-designing the intervention, we believe it is also appropriate for the co-design team to help identify meaningful metrics to evaluate the impact of the designed intervention. While we have identified a means of evaluating team performance (using the ATPI or the TCI), there are likely other measures that will be deemed appropriate based on the final content of the intervention. Given the challenges of change implementation and measurement in a complex system with multiple confounding factors, ${ }^{32}$ multiple kinds of data will be required to enable a thorough and rich assessment of team performance and to understand the impact of the intervention. ${ }^{28}$ Qualitative work at each phase of the research will help us to understand how the intervention is impacting on team working, team performance, patient safety and staff engagement.

Despite the significant changes in the Irish healthcare system in recent years, there is a paucity of research on 
these organisational changes and reconfigurations, which were not necessarily evidence-based. ${ }^{2}$ A key strength of this research is that the engagement of healthcare teams in identifying leadership needs throughout the research will ensure that the intervention is designed collaboratively, thus ensuring its relevance and increasing the likelihood that it will be successful in ultimately improving team performance and enhancing patient safety cultures.

The engagement of hospital staff and clinical leaders as stakeholders and participants will enable the rapid scaling up of the intervention should it prove successful. The institutional partners in this research are University College Dublin, Ireland East Hospital Group, Health Service Executive and The King's Fund, UK. It is intended that each research partner will use its existing networks and partnerships to discuss and disseminate findings; thus, the influence of this study will be on the Irish health system and on the National Health Service in the UK, where staff development in collective leadership is also taking place.

\section{Author affiliations \\ ${ }^{1}$ School of Nursing, Midwifery and Health Systems, Health Sciences Centre, University College Dublin, Dublin, Ireland \\ ${ }^{2}$ Transformation Office, Mater Misericordiae University Hospital, Dublin, Ireland ${ }^{3}$ Division of Quality and Patient Safety, Health Service Executive, Naas, Kildare, Ireland \\ ${ }^{4}$ Our Lady of Lourdes Hospital, Drogheda, Ireland \\ ${ }^{5}$ School of Psychology and Centre for Innovative Human Systems, Trinity College, Dublin, Ireland}

Contributors EMA was responsible for the conceptual design of the research. ADB, MW, MOS, ROD, UC, SMG, JF, SC and NMD were involved with revisions to the design of the research and were involved in the drafting of this manuscript. All authors have read and approved the final manuscript.

Funding This work is funded by the Irish Health Research Board, grant reference number RL-2015-1588. This research is also supported by the Health Service Executive.

Competing interests None declared.

Ethics approval Favourable ethical opinion for the research has been obtained from the University College Dublin Research Ethics Committee (ref: HREC-LS-16-116397/LS-16-20).

Provenance and peer review Not commissioned; externally peer reviewed.

Open Access This is an Open Access article distributed in accordance with the Creative Commons Attribution Non Commercial (CC BY-NC 4.0) license, which permits others to distribute, remix, adapt, build upon this work non-commercially, and license their derivative works on different terms, provided the original work is properly cited and the use is non-commercial. See: http://creativecommons.org/ licenses/by-nc/4.0/

(C) Article author(s) (or their employer(s) unless otherwise stated in the text of the article) 2017. All rights reserved. No commercial use is permitted unless otherwise expressly granted.

\section{REFERENCES}

1. World Health Organization. The world health report 2000: health systems: improving performance: World Health Organization, 2000.

2. McAuliffe E. Clinical governance in the Irish health system - a review of progress. Clinical governance: an international journal 2014;19:296-313.

3. Madden D. Building a culture of patient safety. Report of the commission on patient safety and quality assurance. Dublin, Ireland:
Department of Health and Children, 2008. http://health.gov.ie/wpcontent/uploads/2014/03/en_patientsafety.pdf (accessed 1 Aug 2017).

4. O'Shea Y. Clinical Directorates in the Irish Health Service: Managing Resources and Patient Safety: Orpen Press, 2009.

5. Higgins JR. The establishment of hospital groups as a transition to independent hospital trusts. Department of Health and Children, Dublin, Ireland 2013.

6. West M, Armit K, Loewenthal L, et al; Leadership and leadership development in healthcare: the evidence base. London: The Kings Fund, 2015.

7. Kaufman G, McCaughan D. The effect of organisational culture on patient safety. Nurs Stand 2013;27:50-6.

8. West M, Dawson J, Admasachew L, et al. NHS staff management and health service quality. London: Department of Health, 2011.

9. McKee L. Understanding the dynamics of organisational culture change: creating safe places for patients and staff: University of Aberdeen;, 2010.

10. D'Innocenzo L, Mathieu JE, Kukenberger MR. A meta-analysis of different forms of shared leadership-team performance relations. Journal of Management 2014:0149206314525205.

11. Friedrich TL, Vessey WB, Schuelke MJ, et al. A framework for understanding collective leadership: The selective utilization of leader and team expertise within networks. Leadersh $Q$ 2009;20:933-58.

12. Wang D, Waldman DA, Zhang Z. A meta-analysis of shared leadership and team effectiveness. J Appl Psychol 2014;99:181-98.

13. West M, Eckert R, Steward K, et al. Developing collective leadership for health care. London: The King's Fund, 2014.

14. Higgins JR. The establishment of hospital groups as a transition to independent hospital trusts: Department of Health, 2013. Available from. http://health.gov.ie/wp-content/uploads/2014/03/Full_ Presentation.pdf (accessed 2 Mar 2017).

15. Borgatti SP, Mehra A, Brass DJ, et al. Network analysis in the social sciences. Science 2009;323:892-5.

16. Blanchet $K$, James $P$, do Hto. How to do (or not to do)... a social network analysis in health systems research. Health Policy Plan 2012;27:438-46.

17. Borgatti SP, Everett MG, Freeman LC. Ucinet for Windows: Software for Social Network Analysis. 6 ed. Harvard, MA: Analytic Technologies, 2002.

18. Wasserman S, Faust K. Social network analysis: Methods and applications: Cambridge university press, 1994.

19. Chambers D, Wilson P, Thompson C, et al. Social network analysis in healthcare settings: a systematic scoping review. PLOS One 2012;7:e41911.

20. DeRue DS. Adaptive leadership theory: Leading and following as a complex adaptive process. Res Organ Behav 2011;31:125-50.

21. Contractor NS, DeChurch LA, Carson J, et al. The topology of collective leadership. Leadersh Q 2012;23:994-1011.

22. Contractor NS, Wasserman S, Faust K. Testing multi theoretical multilevel hypotheses about organizational networks: An analytic framework and empirical example. Acad Manage Rev 2006;31:681-703.

23. Braun V, Clarke V. Using thematic analysis in psychology. Qual Res Psychol 2006;3:77-101.

24. Flanagan JC. The critical incident technique. Psychol Bull 1954;51:327-58.

25. Hiller NJ, Day DV, Vance RJ. Collective enactment of leadership roles and team effectiveness: A field study. Leadersh Q 2006;17:387-97.

26. Dawson JF, West MA, Markiewicz L, eds. Aston Team Performance Inventory: management set: ASE, 2006.

27. Anderson NR, West MA. Measuring climate for work group innovation: development and validation of the team climate inventory. J Organ Behav 1998;19:235-58.

28. Tesluk P, Mathieu JE, Zaccaro SJ, et al. Task and aggregation issues in the analysis and assessment of team performance. Team performance assessment and measurement: Theory, methods, and applications, 1997:197-224.

29. Craig P, Dieppe P, Macintyre S, et al. Developing and evaluating complex interventions: the new Medical Research Council guidance. BMJ 2008;337:a1655.

30. Craig P, Dieppe P, Macintyre S, et al. Developing and evaluating complex interventions. Medical Research Council, UK 2011.

31. Goodyear-Smith F, Jackson C, Greenhalgh T. Co-design and implementation research: challenges and solutions for ethics committees. BMC Med Ethics 2015;16:1):1.

32. Plsek PE, Greenhalgh T. The challenge of complexity in health care. British Medical Journal 2001;323:625. 